\title{
Turing-Computability of Solution of Hirota Equation
}

\author{
Dianchen $\mathrm{Lu}^{1, \mathrm{a}}$ and Liming $\mathrm{Fu}^{1, \mathrm{~b}}$
}

\author{
${ }^{1}$ Faculty of Science, Jiangsu University, Zhenjiang Jiangsu, 212013,P.R.China

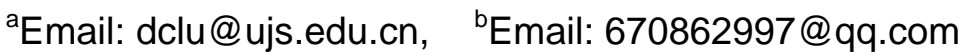

\begin{abstract}
Keywords: Hirota equation, Solution operation, Turing computability, TTE theory, Duhamel principle,

Abstract. In this paper, we mainly discuss the Turing-computable of the Solution operation of Hirota Equation. Firstly, we prove the existence and uniqueness of the solution operator of equation by principle of contraction mapping, and acclaim that its local solution is Turing-Computable in use of TTE theory. By computable functions constructing, we extend the solution from the internal to the entire space. Then the solution of this equation is Turing-computable. The result enlarges the application in computing differential equations on digital computers.
\end{abstract}

\section{Introduction}

Differential equation is an important mathematical model of real problems, but not all have good properties of solutions of differential equations. In reality, we are concerned about how the solution of this equation is calculated. As a result, the computability of solutions for operator becomes a key issue in the study. K.Weihrauch and N.Zhong[1-2] have studied the computability of the solution operator of the wave equation and KDV equation. Dianchen $\mathrm{Lu}$ and others have studied the computability of the nonlinear Kawahara equation[3]. In this paper, we will study the initial problem of Hirota equation:

$$
\begin{cases}\partial_{t} u+i \alpha \partial_{x}^{2} u+\beta \partial_{x}^{3} u+\mu \partial_{x}\left(|u|^{2} u\right)+i \gamma|u|^{2} u=0, & x, t \in R, \\ u(x, 0)=u_{0}(x), & x \in R\end{cases}
$$

where $\alpha, \beta(\mu, \gamma)$ are real (complex) constants and $\alpha \beta \neq 0, u$ is complex valued function. Hirota Equation (1) is a typical model in mathematical physics, which encompasses the well-know nonlinear Schrödinger equation. Hasegawa and Kodama [4-5] proposed (1) as a model for propagation of pulse in optical fiber.

The Cauchy problem of (1) changes as follows if $\mu=0$,

$$
\left\{\begin{array}{l}
\partial_{t} u+i \alpha \partial_{x}^{2} u+\beta \partial_{x}^{3} u+i \gamma|u|^{2} u=0, x, t \in R, \\
u(x, 0)=u_{0}(x), \quad x \in R .
\end{array}\right.
$$

The structure of the article is that: In Part 2, we mainly introduce some basic definitions and lemmas a, which are relevant to the proof of Part3; In Part 3, we prove the main theorem of the paper mainly.

\section{Preliminaries}

Lemma1 $^{[1]}$ (type conversion) Let $\delta_{i}: \subseteq \sum^{\omega} \rightarrow X_{i}$ be a representation of the set $\quad X_{i} \quad, \quad f_{i}: \subseteq X_{1} \times \cdots \times X_{k} \rightarrow X_{0} \quad$, define $L\left(x_{1}, \cdots, x_{k-1}\right)\left(x_{k}\right):=f\left(x_{1}, \cdots, x_{k}\right)$, then if $f$ is $\left(\delta_{1}, \cdots, \delta_{k}, \delta_{0}\right)$-computable if and only if $L$ is $\left(\delta_{1}, \cdots, \delta_{k-1},\left[\delta_{k} \rightarrow \delta_{0}\right]\right)$-computable .

Lemma $2^{[1]}$ Let $\gamma: \subseteq Y \rightarrow M$ and $\gamma^{\prime}: \subseteq Y \rightarrow M^{\prime}$ are two representations, $v_{N}$ is admissible representastion of $N$.Then we have the following propositions: 
1) If $f: \subseteq M \rightarrow M^{\prime}$ is $\left(\gamma, \gamma^{\prime}\right)$-computable, then $f^{\prime}: \subseteq N \times M^{\prime} \times M \rightarrow M^{\prime}$ is $\left(v_{N}, \gamma^{\prime}, \gamma, \gamma^{\prime}\right)$ computable.

We define a function $g^{\prime}: \subseteq N \times M \rightarrow M^{\prime}$ as following:

$$
g^{\prime}(0, x)=f(x), g^{\prime}(n+1, x)=f^{\prime}\left(n, g^{\prime}(n, x), x\right),
$$

Where $x \in M, n \in N$,then $g^{\prime}$ is $\left(v_{N}, \gamma, \gamma^{\prime}\right)$ - computable.

2) Assume that $h: \subseteq M \rightarrow M$ is $(\gamma, \gamma)$ - computable and define a function

$$
H: \subseteq N \times M \rightarrow M: \quad H(0, x)=x, H(n+1, x)=h \circ H(n, x)=h^{n+1}(x) .
$$

So, the function $H$ is $\left(v_{N}, \gamma, \gamma\right)$-computable.

Definition $1^{[6]}$ For $\forall s, b \in R$, the Bourgain space $X_{s, b}$ is the complete space about Schwartz space $S\left(R^{2}\right)$ with the following norm: $\|u\|_{X_{s, b}}=\left\|\langle\xi\rangle^{S}\left\langle\tau-\beta \xi^{3}-\alpha \xi^{2}\right\rangle^{b} F u\right\|_{L_{L^{2}}^{2}}$ or

$\|\bar{u}\|_{\bar{X}_{s, b}}=\left\|\langle\xi\rangle^{S}\left\langle\tau-\beta \xi^{3}+\alpha \xi^{2}\right\rangle^{b} F \bar{u}\right\|_{L_{\xi}^{2} L_{\tau}^{2}}$

where $\langle\bullet\rangle=(1+|\bullet|),\|u\|_{X_{s, b}}=\|\bar{u}\|_{\bar{X}_{s, b}}$.

Lemma $3^{[6]}$ If $s \geq-\frac{1}{4}, \frac{1}{2}<b<\frac{7}{12}, b^{\prime}>\frac{1}{2}$. Then

$\left\|u_{1} u_{2} \overline{u_{3}}\right\|_{X_{s, b-1}} \leq C\left\|u_{1}\right\|_{X_{s, b}}\left\|u_{2}\right\|_{X_{s, b^{\prime}}}\left\|u_{3}\right\|_{X_{s, b^{\prime}}}$

Let $\quad \psi \in C_{0}^{\infty}(R) \quad$ with $\quad \psi=1 \quad$ on $\quad\left[-\frac{1}{2}, \frac{1}{2}\right]$ and sup $p \psi \subset[-1,1]$. We denote $\psi_{\delta}(\bullet)=\psi\left(\delta^{-1}(\bullet)\right)$ for some non-zero $\delta \in R$.

Lemma $4^{[6]}$ If $s \geq-\frac{1}{4}, \frac{1}{2}<b<b^{\prime}<1,0<\delta \leq 1$. Then

$$
\begin{aligned}
& \left\|\psi_{\delta}(t) S(t) \varphi\right\|_{X_{s, b}} \leq C \delta^{\frac{1}{2}-b}\|\varphi\|_{H^{S}}, \\
& \left\|\psi_{\delta}(t) F\right\|_{X_{s, b-1}} \leq C \delta^{b^{\prime}-b}\|F\|_{X_{s, b^{\prime}-1}} \\
& \left\|\psi_{\delta}(t) \int_{0}^{t} S(t-\tau) F(\tau) d \tau\right\|_{X_{s, b}} \leq C \delta^{\frac{1}{2}-b}\|F\|_{X_{s, b-1}}, \\
& \left\|\psi_{\delta}(t) \int_{0}^{t} S(t-\tau) F(\tau) d \tau\right\|_{L_{t}^{\infty} H_{x}^{s}} \leq C \delta^{\frac{1}{2}-b}\|F\|_{X_{s, b-1}}
\end{aligned}
$$

Lemma $5^{[6]}$ If $s \geq-\frac{1}{4}, \frac{1}{2}<b<b^{\prime}<1,0<\delta \leq 1$. Then

$$
\left\|u^{2}|u-| v^{2} \mid v\right\|_{X_{s, b}} \leq \delta^{b^{\prime}-b}\left(\|u\|_{X_{s, b}}^{2}+\|v\|_{X_{s, b}}^{2}\right)\|u-v\|_{X_{s, b}} .
$$

\section{Main Result}

From the problem (2), we establish a nonlinear map $K_{R}: H^{s} \rightarrow C\left(R ; H^{s}(R)\right)$, which translate the initial data $u_{0}$ to the solution $u$. The map $K_{R}$ is the solution operator of the problem (2).

Theorem $1 \forall t \in R$, when $s>-\frac{1}{4}$, the solution operator of the problem $K_{R}: H^{s} \rightarrow C\left(R ; H^{s}(R)\right)$ is $\left(\delta_{H^{s}},\left[\rho \rightarrow \delta_{H^{s}}\right]\right)$-computable.

Proof We can get problem (2) equivalent integral equation by the Duhamel principle: 


$$
u(x, t)=S(t) u_{0}-i \int_{0}^{t} S\left(t-t^{\prime}\right) \gamma|u|^{2}|u|\left(t^{\prime}\right) d t^{\prime},
$$

where $S(t)=F_{X}^{-1} e^{i t\left(\alpha \xi^{2}+\beta \xi^{3}\right)} F_{X}, F_{X}$ represents fourier transform.

For $u_{0} \in H^{s}\left(s \geq-\frac{1}{4}\right)$, define operator

$$
\begin{aligned}
& G\left(u, u_{0}, t\right)=\psi_{1}(t)\left(S(t) u_{0}-i \int_{0}^{t} S\left(t-t^{\prime}\right) \psi_{\delta}\left(t^{\prime}\right) \gamma|u|^{2} u\left(t^{\prime}\right) d t^{\prime}\right), \\
& \bar{G}\left(u, u_{0}\right)(t):=G\left(u, u_{0}, t\right) .
\end{aligned}
$$

According the lemma 3.2 in [1], it is easy way to prove the operator $G$ is $\left(\left[\rho \rightarrow \delta_{s}\right], \delta_{s}, \rho, \delta_{s}\right) \quad$-computable. By lemma 1 , the operator $\bar{G}$ is $\left(\left[\rho \rightarrow \delta_{s}\right], \delta_{s},\left[\rho \rightarrow \delta_{s}\right]\right)$-computable.

Define function $v: S(R) \times N \rightarrow C(R ; S(R))$,

$$
v\left(u_{0}, 0\right)=\bar{G}\left(0, u_{0}\right), v\left(u_{0}, j+1\right)=\bar{G}\left(v\left(u_{0}, j\right), u_{0}\right) .
$$

By Lemma $2 v$ is $\left(\delta_{s}, v_{N},\left[\rho \rightarrow \delta_{s}\right]\right)$-computable.

Let $\omega(x, t)=u\left(x, t_{0}+t\right), t \in[0, T], t_{0} \geq 0$.

If $u\left(x, t_{0}\right)=\varphi(x)$, then

$$
\begin{cases}\partial_{t} \omega+i \alpha \partial_{x}^{2} \omega+\beta \partial_{x}^{3} \omega+i \gamma|\omega|^{2} \omega=0, & x, t \in R, \\ \omega(x, 0)=\varphi(x), & x \in R .\end{cases}
$$

We assume that the initial value $\varphi \in H^{s}(R)$ is given by a $\tilde{\delta}_{H^{s}}$-name, i.e., $p=<p_{0}, p_{1}, \cdots>$ which is obtained by $\delta_{s c}\left(p_{i}\right)=\varphi_{i}$ and $\left\|\varphi_{n}-\varphi\right\|_{s} \leq 2^{-n-2}$. For $\forall k \in N$, there exists computable $n_{k}$ satisfying $\left\|\varphi_{n_{k}}-\varphi\right\|_{H^{s}} \leq 2^{-n_{k}-2} \leq 2^{-k-2}$.

We define functions

$$
\omega_{n}^{0}:=\bar{G}\left(0, \varphi_{n}\right), \omega_{n}^{j+1}:=\bar{G}\left(\omega_{n}^{j}, \varphi_{n}\right) .
$$

It is easy to prove $\omega_{n}^{j} \rightarrow \omega_{n} \quad(j \rightarrow \infty)$, there $\omega_{n}$ satisfies the following integral equation:

$$
\begin{cases}\frac{\partial \omega_{n}}{\partial t}+i \alpha \frac{\partial^{2} \omega_{n}}{\partial x^{2}}+\beta \frac{\partial^{3} \omega_{n}}{\partial x^{3}}+i \gamma\left|\omega_{n}\right|^{2} \omega_{n}=0, & x, t \in R, \\ \omega_{n}(x, 0)=\mu_{n}(x), & x \in R .\end{cases}
$$

Since $\lim _{j \rightarrow \infty} \omega_{n}^{j}=\omega_{n}(j \rightarrow \infty)$, we can select suitable integer $n_{k}, j_{k}$ to contract a sequence $\left\{\omega_{n_{k}}^{j_{k}}\right\}_{k \in N}$, satisfying $\left\|\omega_{n_{k}}^{j_{k}}-\omega_{n_{k}}\right\|_{s} \leq 2^{-k-1}$.Then $\left\{\omega_{n_{k}}^{j_{k}}\right\}_{k \in N}$ is computable sequence.

In following, we prove $\left\{\omega_{n_{k}}^{j_{k}}\right\}_{k \in N}$ fastly converges to $\omega$.

From (3)-(8), we obtain 


$$
\begin{aligned}
& \quad\left\|\omega_{n_{k}}-\omega\right\|_{X_{s, b}}=\left\|\psi_{1}(t) S(t)\left(\varphi_{n}(x)-\varphi(x)\right)-\psi_{1}(t) i \int_{0}^{t} S\left(t-t^{\prime}\right) \psi_{\delta}\left(t^{\prime}\right) \gamma\left(|\omega|^{2} \omega-\left|\omega_{n_{k}}\right| \omega_{n_{k}}\right) d t^{\prime}\right\|_{X_{s, b}} \\
& \leq\left\|\psi_{1}(t) S(t)\left(\varphi_{n}(x)-\varphi(x)\right)\right\|_{X_{s, b}}+\left\|\psi_{1}(t) i \int_{0}^{t} S\left(t-t^{\prime}\right) \psi_{\delta}\left(t^{\prime}\right) \gamma\left(|\omega|^{2} \omega-\left|\omega_{n_{k}}\right| \omega_{n_{k}}\right) d t^{\prime}\right\|_{X_{s, b}} \\
& \leq C_{1}\left\|\varphi_{n}(x)-\varphi(x)\right\|_{X_{s, b}}+C \delta^{\frac{1}{2}-b}|\gamma|\left\||\omega|^{2} \omega-\left|\omega_{n_{k}}\right| \omega_{n_{k}}\right\|_{X_{s, b}-1} \\
& \leq C_{1}\left\|\varphi_{n}(x)-\varphi(x)\right\|+C|\gamma| \delta^{b^{\prime}-b}\left(\|\omega\|_{X_{s, b}}^{2}+\left\|\omega_{n_{k}}\right\|_{X_{s, b}}^{2}\right)\left\|\omega-\omega_{n_{k}}\right\|_{X_{s, b}} \\
& \leq C_{1} 2^{-k-2}+4 C C_{r}^{2}|\gamma| \delta^{b^{\prime}-b}\left\|\omega-\omega_{n_{k}}\right\|_{X_{s, b}}
\end{aligned}
$$

Choosing sufficient small $\delta$ such that $\frac{C_{1}}{1-4 C r C_{4} \delta^{b^{\prime}-b}}<2$, then

$$
\left\|\omega_{n_{k}}-\omega\right\|_{X_{s, \frac{1}{2}}} \leq 2^{-k-1}
$$

Thus $\left\|\omega_{n_{k}}^{j_{k}}-\omega\right\|_{X_{s, \frac{1}{2}}} \leq\left\|\omega_{n_{k}}^{j_{k}}-\omega_{n_{k}}\right\|_{X_{s, \frac{1}{2}}}+\left\|\omega_{n_{k}}-\omega\right\|_{X_{s, \frac{1}{2}}} \leq 2^{-k-1}+2^{-k-1} \leq 2^{-k}$

Then we have proved $\left\{\omega_{n_{k}}^{j_{k}}\right\}_{k \in N}$ fastly converges to $\omega$ and $\omega$ is computable.

We have known $\left\{\omega_{n_{k}}^{j_{k}}\right\}_{k \in N}$ is computable sequence, if $\delta_{S}\left(q_{k}\right)=\omega_{n_{k}}^{j_{k}}(t)$,then $\tilde{\delta}_{H^{s}}\left\langle q_{0}, q_{1}, \cdots\right\rangle=\omega(t)$, i.e., $\left\langle q_{0}, q_{1}, \cdots\right\rangle$ is the $\tilde{\delta}_{H^{s}}$-name of $\omega(t)$. Hence the solution $\omega$ of the initial problem (9) is computable on $t \in[0, T]$.

We define a $\left(\rho, \tilde{\delta}_{H^{s}}, \rho, \tilde{\delta}_{H^{s}}\right)$-computable map $I:\left(t_{0}, u_{0}, t\right) \rightarrow u(t), t \in\left[t_{0}, t_{0}+T\right]$

where $\omega\left(t_{0}\right)=u_{0}, \omega(t)$ is the solution of the initial problem (2) on $t \in\left[t_{0}, t_{0}+T\right]$.

Then we prove the solution $u(n \cdot T)$ is computable. The function $H: H(\phi, n)=u(n T)$ defined by

$$
H\left(u_{0}, 0\right)=u_{0}, H\left(u_{0}, n+1\right)=I\left(n T, H\left(u_{0}, n\right),(n+1) T\right) .
$$

It is easy to prove the function $H$ is computable since $H$ is derived by primitive recursion from computable function $I$.

In the end, we prove $u(t)$ is computable. Let $n \cdot T \leq t \leq(n+1) \cdot T$, we firstly compute $u(n \cdot T)$, then compute $I(n T, u(n T), t)$, so $u(t)=I(n T, u(n T), t)$ is computable.

In this way, we have get the computable solution on $t \in R$. For $\forall t \in R, s \geq-\frac{1}{4}$, the solution operator $K_{R}: H^{s} \rightarrow C\left(R ; H^{s}(R)\right)$ of Hirota equation(2) is $\left(\delta_{s},\left[\rho \rightarrow \delta_{s}\right]\right)$-computable .

Similarly, we can prove the solution operator of Hirota equation is computable.

\section{Acknowledgements}

The work was supported by the National Nature Science Foundation of China (No. 61070231), and Natural Science Foundation of Jiangsu Province (BK20140525).

\section{References}

[1] Weihrauch.K, Zhong.N. Computing the solution of the Korteweg-de Vries equation with arbitrary precision on Turing machines. Theoret.Comput.Sci,V332, 2005, pp.337-366. 
[2] Weihrauch.K, Zhong.N. Is wave propagation computable or can wave computers beat the Turing machine?. Proc. London Math. Soc., V85 (2),2002, pp.312-332.

[3] Dianchen.L, Jiaxin.G. Computable analysis of the solution of the Nonlinear Kawahara equation. IJCSET,V2,2012, Issue 4, pp.1059-1064.

[4] Hasegawa.A, Kodama.Y. Nonlinear pulse propagation in a monomode dielectric guide. IEEE Journal of Quantum Electronics,V23,1987, pp.510-524.

[5] Kodama.Y. Optical solitons in a monomod fiber. Journal of Statistical phys,V39, 1985, pp.597-614.

[6] Zhaohui.H, Yueling.J. Well-posedness for the cauchy problem to the Hirota equation in sobolev spaces of negative indices. Chin.Ann.Math., V26B, 2005, pp.75-88.

[7] Caixia.S. Modified KdV equation and D-S posedness and ill-posed problems. Chinese Academy of Engineering Physics, 2006. 